\title{
Paediatric otitis media at a primary healthcare clinic in South Africa
}

\author{
L Biagio, ${ }^{1}$ MComMPath; D W Swanepoel, ${ }^{1.3} \mathrm{PhD} ;$ C Laurent, ${ }^{1,4} \mathrm{MD}$, PhD; T Lundberg, ${ }^{5} \mathrm{MD}$ \\ ${ }^{1}$ Department of Speech-Language Pathology and Audiology, University of Pretoria, South Africa \\ ${ }^{2}$ Ear Sciences Centre, School of Surgery, University of Western Australia, Nedlands, Australia \\ ${ }^{3}$ Ear Science Institute Australia, Subiaco, Australia \\ ${ }^{4}$ ENT Unit, Department of Clinical Science, Umeå University, Sweden \\ ${ }^{5}$ Family Medicine, Department of Public Health and Clinical Medicine, Umeå University, Sweden
}

Corresponding author: L Biagio (leigh.biagio@up.ac.za)

\begin{abstract}
Background. No published studies on the prevalence of paediatric otitis media at primary healthcare clinics (PHCs) in South Africa (SA) are available.

Objective. To examine the point prevalence of otitis media in a paediatric population in a PHC in Johannesburg, SA, using otomicroscopy. Methods. A sample of 140 children aged 2 - 16 years (mean 6.4; 44.1\% females) were recruited from patients attending the PHC. Otomicroscopy was completed for each of the participants' ears by a specialist otologist using a surgical microscope.

Results. Cerumen removal was necessary in $36.0 \%$ of participants (23.5\% of ears). Otitis media with effusion was the most frequent diagnosis (16.5\%). Chronic suppurative otitis media (CSOM) was diagnosed in $6.6 \%$ of children and was the most common type of otitis media in participants aged 6 - 15 years. Acute otitis media was only diagnosed in the younger 2 - 5 -year age group (1.7\%). Otitis media was significantly more prevalent among younger (31.4\%) than older children (16.7\%).

Conclusion. CSOM prevalence, as classified by the World Health Organization, was high. Consequently diagnosis, treatment and subsequent referral protocols may need to be reviewed to prevent CSOM complications.
\end{abstract}

S Afr Med J 2014;104(6):431-435. DOI:10.7196/SAMJ.7534

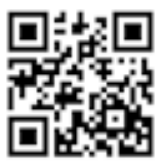

Otitis media is a pervasive childhood disease posing significant healthcare challenges. ${ }^{[1]}$ Estimates suggest that $80 \%$ of children will have at least one episode of acute otitis media (AOM) before 3 years of age. ${ }^{[2]} \mathrm{A}$ global incidence study reported an AOM incidence rate of $10.9 \%{ }^{[3]}$ The true incidence of otitis media with effusion (OME) is difficult to estimate as the disorder is by definition asymptomatic. Furthermore, most screening studies determine the presence of middle-ear fluid without differentiating between AOM and OME ${ }^{[4]}$ An analysis of previous studies estimated the point prevalence of middle-ear effusion on screening as $20.0 \% \cdot{ }^{[4]}$ The global incidence of the most severe form of otitis media, chronic suppurative otitis media (CSOM), is $4.8 \%$. CSOM is estimated to contribute to more than half of the global burden of hearing impairment. ${ }^{[5]}$

The burden and population demographics of otitis media differ greatly between developed and developing regions. India and subSaharan Africa (SSA) account for most deaths from complications arising from otitis media. ${ }^{[5]}$ The incidence of AOM in SSA, South Asia and Oceania is two- to eight-fold higher than in the rest of the world, ${ }^{[3]}$ with the aboriginal population demonstrating the highest incidence. ${ }^{[4]}$ SSA has the second-highest incidence of CSOM. ${ }^{[3]}$

The epidemiology of otitis media, and reasons for regional differences in incidence and prevalence are complex, with risk factors involving multiple host-related factors (age, gender, race, allergy, immune competence specifically related to HIV status, malnutrition, craniofacial abnormalities, genetic predisposition) and environmental factors (upper respiratory infection, seasonality, daycare, presence of siblings, tobacco smoke exposure, breastfeeding, socioeconomic status). ${ }^{[1,3,4,6]}$ HIV-positive children are more prone to, and more severely affected by, otitis media than immunocompetent children. ${ }^{[7]}$
An estimated 3 million of the 3.3 million children worldwide who are HIV-positive (0 - 14 years of age) live in SSA.$^{[8]}$ Of the SSA countries, South Africa (SA) has the second-highest prevalence of new HIV infections in children ${ }^{[9]}$ HIV status is therefore likely to be important factor in the epidemiology of otitis media in SA.

Despite the diverse risk factors and influences, otitis media is largely preventable, and can be effectively managed through medical and surgical approaches. ${ }^{[6]}$ However, knowledge of the prevalence of otitis media, especially of the most severe form of the disease CSOM - is important in determining treatment protocols. ${ }^{[6]}$ In a community where CSOM prevalence is low, the disease will generally resolve without treatment or complications. ${ }^{[6]}$ However, early medical intervention is indicated in communities where CSOM prevalence rates are $>4.0 \%$, which is considered a high-risk population. ${ }^{[5,6]}$

The World Health Organization (WHO) has classified the prevalence of CSOM in Africa among children and adults as high, estimated to be between $3.0 \%$ and $6.0 \%{ }^{[5]}$ Estimates of otitis media in SA, which were included in recent global prevalence studies completed by Acuin ${ }^{[5]}$ and Monasta et al. ${ }^{[3]}$ are based on only two studies. ${ }^{[10,11]}$ One additional study provided an indication of the prevalence of only OME in SA. ${ }^{[12]}$ Estimates of the point prevalence of otitis media in SA rural populations varied from $6.5 \%$ to $18.0 \%{ }^{[11,12]} \mathrm{OME}$ was reported most frequently by all studies (paediatric prevalence of $3.8-12.0 \%$ ), with CSOM seen in $0.3-6.0 \%$ of the paediatric population. ${ }^{[10-12]}$ However, variations in CSOM definition make comparison between studies difficult.

Previous SA studies investigating otitis media prevalence selected rural populations, characterised by many of the poor socioeconomic conditions associated with otitis media, but focused on school-aged children ${ }^{[10,11]}$ as opposed to younger preschool children who are more prone to otitis media. ${ }^{[4]}$ Otoscopy, rather than otomicroscopy, was 
used previously to diagnose middle-ear pathology. Otomicroscopy, however, offers greater diagnostic sensitivity and specificity than either otoscopy or pneumatic otoscopy, ${ }^{[13]}$ and is therefore likely to provide a more accurate diagnosis, and classification, of otitis media. No studies on otitis media prevalence have been performed at primary healthcare clinics (PHCs) in SA. The current study therefore employed otomicroscopy to ascertain the point prevalence of otitis media in a paediatric population in an SA PHC.

\section{Methods}

\section{Study population}

Witkoppen Health and Welfare Centre (WHWC) is a PHC that provides services to the Diepsloot community north of Johannesburg, SA.

Diepsloot is a densely populated settlement consisting of government-subsidised housing, brick houses built by landowners, and shacks fashioned from scrap metal, wood, plastic and cardboard. ${ }^{[14]}$ Estimates suggest that $>90 \%$ of the $>150000$ population is unemployed, with many families lacking access to basic services such as running water, sewage and rubbish removal.

WHWC serves as a specialist centre for HIV and tuberculosis (TB) treatment. In 2012, the clinic had 95521 patient visits. Of the children $(<14$ years) who attended the clinic in 2012, and whose caregivers consented, $4.0 \%$ tested positive for HIV.

A consecutive sample of 140 children aged 2 - 16 years (range 2 15.8 ; mean ( \pm standard deviation $(\mathrm{SD})) 6.4( \pm 3.5)$; $44.1 \%$ females) were recruited from registered patients of WHWC.

The participants were recruited from the entire paediatric population attending the clinic for any purpose, whether for a routine clinic appointment or for chronic or acute treatment. Caregivers were required to provide written consent after being informed (verbally and in writing) of the study objectives and methods. Although the typical annual paediatric HIV prevalence among patients of WHWC who consented to HIV testing was known, HIV status for the individual study participants was not recorded because ethical clearance did not allow for this. Caregivers and children were interviewed immediately prior to otomicroscopy to obtain biographical information and history of earache, ear discharge or hearing loss during the 2 weeks prior to participation in the study.

The investigation was conducted following approval from the Research Ethics Committee of the Faculty of Humanities, University of Pretoria.

\section{Data collection}

Otomicroscopy was completed for each ear of the participants by a specialist otologist using a Leica M525 F40 surgical microscope. Observations regarding ear canal obstruction, presence of secretion, tympanic membrane patency, translucency and position, as well as the concluding diagnosis were documented. On-site data collection continued over the course of 2 weeks.

The diagnosis and classification of the types of otitis media were based on: (i) clinical data of otalgia and otomicroscopic findings of opacity and bulging of the intact tympanic membrane for $\mathrm{AOM}^{;[15]}($ ii) a wet, swollen and contourless eardrum for perforated AOM; (iii) evidence of seromucoid effusion in the middle ear (completely filled or air-fluid level or bubbles), with an intact tympanic membrane without symptoms of acute infection for OME; ${ }^{[15]}$ and (iv) evidence of a perforation or cholesteatoma with or without purulent discharge for CSOM. ${ }^{[5]}$

\section{Data analysis}

Otomicroscopic examinations could be completed for 136 participants (272 ears) (four participants failed to co-operate during otomicroscopy). Descriptive statistics were used to determine the frequency with which the caregivers reported otological symptoms, the presence of cerumen, and otological status of otitis media for the age groups 2 - 5 years and 6 - 15 years. The two age groups were delineated to represent preschool children (aged 2 - 5 years) and children attending school (aged 6 15 years). Descriptive statistics on otological status were presented for participants and ears, respectively. A participant was classified as 'normal' when an otological diagnosis of 'normal' was made on otomicroscopy in both ears. When a diagnosis could not be made by otomicroscopy due to partial or complete obstruction of the external ear canal, the ear was classified as 'undetermined'. If a participant had 'undetermined' status in one or both ears, the participant was classified as 'undetermined. Descriptive statistics on otological status were also completed excluding cases where a diagnosis could not be made in one or both ears. Comparisons of otological status (for data including and excluding 'undetermined' ears) between age groups, gender, and left and right ears were made using Pearson's $\chi^{2}$ tests with $p<0.05$ considered to be significant.

\section{Results}

Table 1 summarises the symptoms and complaints of otological disorders for the 2 weeks prior to evaluation, as disclosed by the participants' caregivers. Caregivers indicated that $7.4 \%$ of participants presented with earache, $5.2 \%$ with discharge and $6.6 \%$ with possible hearing loss. Earache and discharge for participants aged $2-5$ years were reported almost twice as often as for participants aged 6 - 15 years.

Cerumen removal was necessary for $36.0 \%$ of participants $(23.5 \%$ of ears) to obtain a clear view of the tympanic membrane for otomicroscopic diagnosis. Cerumen was removed manually in this study and was halted in the event of any discomfort. Cerumen removal was required for $39.5 \%$ of 2 - 5 -year-old participants $(23.7 \%$ of ears), and for $31.7 \%$ of participants aged $6-15$ years $(23.3 \%$ of ears). Cleaning was not possible for a greater number of participants aged 2 - 5 years (14.5\% of participants) than those aged 6 - 15 years (10.0\% of participants). Table 2 summarises the participants and ears with no, partial and complete obstruction of the tympanic membrane by cerumen (after manual cerumen removal as far as was possible without causing discomfort) at the time of otomicroscopic diagnosis of middle-ear status.

The otological status was reported for both ears and participants (Table 3). Cerumen could not be removed, and otomicroscopic diagnosis of middle-ear status could consequently not be made, in one or both ears of $12.9 \%$ of participants. A diagnosis could not be made in either ear of three participants.

Table 4 summarises the otological status for the paediatric sample but excludes ears where a diagnosis could not be made due to partial or complete cerumen obstruction. Diagnosis by otomicroscopic examination revealed that otitis media was significantly more prevalent for the younger $(31.4 \%) \mathrm{v}$. the older participants (16.7\%; $p=0.034 ; \chi^{2}$ test). OME was the most frequently diagnosed pathology for participants aged 2 - 5 years (23.9\%), while CSOM was most commonly diagnosed for those aged $6-15$ years $(9.3 \%)$. AOM was only diagnosed in the younger participants (3.0\%).

Table 1. Caregiver report of symptoms of otological disorder over 2 weeks prior to otomicroscopy $(N=136)$

\begin{tabular}{llll}
\hline & \multicolumn{2}{c}{ Age (yrs), \% } & \\
\cline { 2 - 3 } & $\mathbf{2 - 5}$ & $\mathbf{6 - 1 5}$ & Total, \% \\
\hline Earache & 9.2 & 5.0 & 7.4 \\
Hearing loss & 2.6 & 11.7 & 6.6 \\
Discharge & 6.6 & 3.3 & 5.2
\end{tabular}


Otitis media was equally distributed between right and left ears. More male participants $(29.2 \%)$ than female participants (18.5\%) presented with otitis media, but the difference was not statistically significant $\left(p>0.05 ; \chi^{2}\right.$ test).

\section{Discussion}

Establishing regional otitis media prevalence rates is important when determining treatment protocols. ${ }^{[6]}$ The point prevalence of OME in

Table 2. Obstruction of the tympanic membrane during otomicroscopic examination

\begin{tabular}{|c|c|c|c|}
\hline & \multicolumn{2}{|c|}{ Age (yrs), \% } & \multirow[b]{2}{*}{ Total, \% } \\
\hline & $2-5$ & $6-15$ & \\
\hline \multicolumn{4}{|l|}{ Subjects } \\
\hline Total, $n$ & 76 & 60 & 136 \\
\hline \multicolumn{4}{|l|}{ Bilateral obstruction } \\
\hline None & 30.3 & 46.7 & 37.5 \\
\hline Partial & 44.7 & 23.3 & 35.3 \\
\hline Complete & 0.0 & 1.7 & 0.7 \\
\hline Partial and complete & 9.2 & 10.0 & 9.6 \\
\hline \multicolumn{4}{|l|}{ Unilateral obstruction } \\
\hline Partial & 13.2 & 11.6 & 12.5 \\
\hline Complete & 2.6 & 6.7 & 4.4 \\
\hline \multicolumn{4}{|l|}{ Ears } \\
\hline Total, $n$ & 152 & 120 & 272 \\
\hline \multicolumn{4}{|l|}{ Obstruction } \\
\hline None & 38.8 & 55.8 & 46.3 \\
\hline Partial & 55.9 & 34.2 & 46.3 \\
\hline Complete & 5.3 & 10.0 & 7.4 \\
\hline
\end{tabular}

the current study population (excluding undetermined participants) was $16.5 \%$, which is the highest in current reports from SA. ${ }^{[10-12]}$ It is also higher than the non-aboriginal OME prevalence in the AsiaPacific region $\left(1.14\right.$ - 13.8\%), ${ }^{[16]}$ but still considerably lower than bilateral OME prevalence for aboriginal children $(31.0 \%)$, who have the highest OME prevalence rate in the world. ${ }^{[16]}$

A higher prevalence of OME for male participants was found in the current study, although it was not statistically significant $(p>0.05$; $\chi^{2}$ test). Previous research on gender differences have reported divergent findings with some demonstrating a statistically significant higher prevalence in male children, while others found no gender differences. ${ }^{[2,4]}$ Our study findings support that of Teele et al., ${ }^{[2]}$ who found that OME was associated with male children. Although HIV status was not assessed in participants enrolled in our study, WHWC reported a new HIV prevalence rate of $4.0 \%$ among children aged $\leq 14$ years in 2012. This is higher than the SA HIV prevalence rate of $2.4 \%$ for babies tested at 6 weeks of age (reported between March 2012 and February 2013). ${ }^{[17]}$ Individuals with HIV are known to be more prone to, and more severely affected by, otitis media than seronegative children. ${ }^{[18]}$ Positive HIV status may therefore have contributed to the OME point prevalence measured, and to that of the more severe form of otitis media, namely CSOM.

In our study, the prevalence of CSOM for the total paediatric sample (excluding undetermined participants) was 6.6\%, which is classified by the WHO as high, ${ }^{[5]}$ and is similar to previous estimates of CSOM prevalence in SSA. ${ }^{[5]}$ The CSOM prevalence of $9.3 \%$ measured among 6 - 15-year-olds in the current study would be rated as the highest prevalence according to the WHO classification system. ${ }^{[5]}$ The higher CSOM prevalence for older children was anticipated as the pathology and sequelae develop from long-term, chronic middle-ear inflammation. Differences in the terminology and definitions used in previous studies make comparisons problematic. Perforations of the tympanic membrane can be associated with either AOM or CSOM. Prescott and Kibel ${ }^{[11]}$ did not report CSOM, but described the prevalence of perforated tympanic membranes in their study (6.0\% of participants), which was comparable

Table 3. Otological status as diagnosed by otomicroscopy

\begin{tabular}{|c|c|c|c|c|c|c|c|}
\hline & \multicolumn{3}{|c|}{ Age (yrs), \% } & \multirow[b]{2}{*}{ Male, \% } & \multirow[b]{2}{*}{ Female, \% } & \multirow[b]{2}{*}{ Left, \% } & \multirow[b]{2}{*}{ Right, \% } \\
\hline & All, \% & $2-5$ & $6-15$ & & & & \\
\hline \multicolumn{8}{|l|}{ Participants } \\
\hline Total, $n$ & 136 & 76 & 60 & 75 & 61 & - & - \\
\hline Normal & 66.9 & 60.5 & 75.0 & 61.4 & 72.1 & - & - \\
\hline Otitis media & 20.6 & 27.7 & 11.7 & 25.3 & 16.4 & - & - \\
\hline $\mathrm{AOM}$ & 1.5 & 2.6 & 0.0 & 1.3 & 1.6 & - & - \\
\hline CSOM & 5.1 & 4.0 & 6.7 & 6.7 & 4.9 & - & - \\
\hline OME & 14.0 & 21.1 & 5.0 & 17.3 & 9.9 & - & - \\
\hline Undetermined & 12.5 & 11.8 & 13.3 & 13.3 & 11.5 & - & - \\
\hline \multicolumn{8}{|l|}{ Ears } \\
\hline Total, $n$ & 272 & 152 & 120 & 150 & 122 & 136 & 136 \\
\hline Normal & 75.8 & 71.1 & 81.6 & 72.0 & 81.4 & 75.7 & 75.7 \\
\hline Otitis media & 16.5 & 21.0 & 10.9 & 19.3 & 11.8 & 17.7 & 15.5 \\
\hline $\mathrm{AOM}$ & 0.7 & 1.3 & 0.0 & 0.7 & 0.8 & 1.5 & 0.0 \\
\hline CSOM & 4.8 & 3.3 & 6.7 & 5.3 & 4.2 & 4.4 & 5.2 \\
\hline OME & 11.0 & 16.4 & 4.2 & 13.3 & 6.8 & 11.8 & 10.3 \\
\hline Undetermined & 7.7 & 7.9 & 7.5 & 8.7 & 6.8 & 6.6 & 8.8 \\
\hline
\end{tabular}


Table 4. Otological status as diagnosed by otomicroscopy excluding participants and ears where a diagnosis could not be made

\begin{tabular}{|c|c|c|c|c|c|c|c|}
\hline & \multirow[b]{2}{*}{ All, \% } & \multicolumn{2}{|c|}{ Age (yrs), \% } & \multirow[b]{2}{*}{ Male, \% } & \multirow[b]{2}{*}{ Female, \% } & \multirow[b]{2}{*}{ Left, \% } & \multirow[b]{2}{*}{ Right, \% } \\
\hline & & $2-5$ & $6-15$ & & & & \\
\hline \multicolumn{8}{|l|}{ Participants } \\
\hline Total, $n$ & 121 & 67 & 54 & 65 & 54 & - & - \\
\hline Normal & 75.2 & 68.6 & 83.3 & 70.8 & 81.5 & - & - \\
\hline Otitis media & 24.8 & 31.4 & 16.7 & 29.2 & 18.5 & - & - \\
\hline $\mathrm{AOM}$ & 1.7 & 3.0 & 0.0 & 1.5 & 1.8 & - & - \\
\hline CSOM & 6.6 & 4.5 & 9.3 & 7.7 & 5.6 & - & - \\
\hline OME & 16.5 & 23.9 & 7.4 & 20.0 & 11.1 & - & - \\
\hline \multicolumn{8}{|l|}{ Ears } \\
\hline Total, $n$ & 251 & 140 & 111 & 137 & 110 & 127 & 124 \\
\hline Normal & 82.1 & 77.1 & 88.3 & 78.8 & 87.3 & 81.1 & 83.1 \\
\hline Otitis media & 17.9 & 22.9 & 11.7 & 21.2 & 12.7 & 18.9 & 16.9 \\
\hline $\mathrm{AOM}$ & 0.8 & 1.4 & 0.0 & 0.7 & 0.9 & 1.6 & 0.0 \\
\hline CSOM & 5.2 & 3.6 & 7.2 & 5.9 & 4.5 & 4.7 & 5.6 \\
\hline OME & 11.9 & 17.9 & 4.5 & 14.6 & 7.3 & 12.6 & 11.3 \\
\hline
\end{tabular}

with the CSOM prevalence of the total sample of our study $(6.6 \%$ of participants). The CSOM prevalence in primary-school children reported in our study and that of Prescott and Kibel ${ }^{[1]}$ is considerably higher than in previous SA studies. ${ }^{[10,12]}$ Formal healthcare institutions, albeit at different levels (primary v. tertiary) of healthcare, were sampled in our study and in that of Prescott and Kibel, ${ }^{[11]}$ which may explain the higher CSOM prevalence than the school populations targeted by Halama et al..$^{[10]}$ and $\mathrm{Nel}$ et al..$^{[12]}$ In a recent study on the otological, audiological and bacteriological findings in children with CSOM in an SA tertiary hospital, HIV infection was present in $54.6 \%$ of participants with $\mathrm{CSOM}^{[19]}$ As WHWC is a specialist HIV centre, the HIV rate among participants may have contributed to a higher CSOM prevalence than the findings of Halama et al. ${ }^{[10]}$ and Nel et al. ${ }^{[12]}$

From the sample of 272 ears, only two ears $(0.8 \%)$ were identified with AOM (1.7\% of participants, excluding those where a diagnosis could not be made). As may be expected, given that AOM prevalence decreases with age, the two participants diagnosed with AOM fell within the 2 - 5-year age group. ${ }^{[2]}$ Previous SA studies did not distinguish between active AOM, OME and 'previous evidence' of $\mathrm{AOM}^{[10-12]} \mathrm{A}$ study performed by Swart et al..$^{[20]}$ on 24305 - 8-yearold children in rural Swaziland reported a lower point prevalence of AOM $(0.007 \%))^{[20]}$ The lower prevalence of AOM reported by Swart et al. ${ }^{[201}$ compared with our study (1.7\%) may be due to their exclusion of children $<5$ years of age. A recent study by Chadha et al. ${ }^{[21]}$ of 15718 children in India reported an AOM point prevalence rate of $0.65 \%$. This is also lower than was found in our study, but children $<5$ years of age were again excluded from Chadha et al.s study.

Timing of data collection and possible seasonal influences on $\mathrm{AOM}$ as a complication of chronic allergic rhinitis may also have played a role in the AOM point prevalence measured. ${ }^{[1,22]}$ However, the expression of allergic rhinitis in SA is mainly that of a persistent disease, especially inland where grass pollens are present for significant periods of time. ${ }^{[2]}$ What was noteworthy was that caregivers of $7.4 \%$ of the participants reported that their children complained of earache within 2 weeks of the assessment. This suggests that caregivers may not seek medical opinion in response to episodic otalgia, but rather adhere to fixed clinic visit schedules. Additionally, the rapid spontaneous recovery rate for AOM $(80 \%$ within 2 - 3 days $)^{[23]}$ means that a point prevalence study such as ours is likely to underestimate the actual occurrence of AOM. ${ }^{[3]}$

Although not the most common finding on otomicroscopy, the presence of complete occlusion of the ear canal due to impacted cerumen was higher $(14.7 \%$ unilateral and bilateral complete obstruction from cerumen) than in the studies by Chadha et al. ${ }^{[2]]}$ and Swart et al. ${ }^{[20]}(7.93 \%$ and $7.5 \%$, respectively). The rate of cerumen impaction was, however, similar to a previous finding for a sample of SA school children, where impacted cerumen was reported in $14 \%$ of paediatric participants. ${ }^{[1]}$

The CSOM point prevalence measured in our study has implications for treatment protocols. In low-risk populations, OME and AOM are conditions that mostly resolve without treatment or complications. ${ }^{[6]}$ Although beyond the scope of the current research, intervention paradigms may have to be reassessed in the light of the categorisation of the high CSOM prevalence in the paediatric population sampled according to WHO criteria. ${ }^{[5]}$ Many of the risk factors to which high rates of CSOM are attributed could be identified in the population sampled, including short-term breastfeeding, overcrowding, poor hygiene, poor nutrition, and exposure to tobacco, wood and charcoal smoke. ${ }^{[4]}$ Caregivers need to be informed of the high prevalence of complications of otitis media among children in the community, and the impact of hearing impairment caused by CSOM, and should be encouraged to seek medical advice for any symptoms of ear disease. Other measures that may reduce the burden of otitis media include routine otological screening of school children and increased referral of children with recurrent ear disease for specialist opinion. ${ }^{[2]}$ Further research using strict CSOM diagnostic criteria is required to determine whether the prevalence rates measured in our study are typical of the experience of PHCs in underserved communities in SA.

\section{Study limitations}

Our study included a smaller sample of participants than previous similar otitis media prevalence studies. ${ }^{[1-12]}$ With larger participant numbers, stratification of the sample across age groups would be possible. Future studies on the prevalence of OME in a paediatric sample at primary healthcare levels in SA would be improved by careful documentation of additional disease and treatment regimens, especially those related to HIV and TB status. 


\section{Conclusion}

No studies on otitis media prevalence have been performed previously at primary healthcare level in paediatric populations in SA. Otitis media was significantly more prevalent among younger $(31.4 \%)$ than older children (16.7\%). We found an OME point prevalence of $16.5 \%$ for children aged between 2 and 16 years with a CSOM prevalence of $6.6 \%$, which is classified by the WHO as high. ${ }^{[5]}$ The lack of timely medical intervention, together with the presence of environmental risk factors for otitis media, may explain the high rate of CSOM identified. With CSOM prevalence rates at a PHC being this high, the diagnosis, treatment and subsequent referral protocols may need to be reviewed to prevent complications.

\section{References}

1. Daly KA, Hoffman HJ, Kvaerner KJ, et al. Epidemiology, natural history, and risk factors: Panel report from the Ninth International Research Conference on Otitis Media. Int J Pediatr Otorhinolaryngol 2010;74(3):231-240. http://dx.doi.org/10.1016/j.ijporl.2009.09.006

2. Teele DW, Klein JO, Rosner B. Epidemiology of otitis media during the first seven years of life in children in greater Boston: A prospective, cohort study. J Infect Dis 1989;160(1):83-94. [http://dx.doi.org/10.1093/ infdis/160.1.83]

3. Monasta L, Ronfani L, Marchetti F, et al. Burden of disease caused by otitis media: Systematic review and global estimates. PloS One 2012;7(4):1-12. [http://dx.doi.org/10.1371/journal.pone.0036226]

4. Casselbrant ML, Mandel EM. Epidemiology. In: Rosenfeld RM, Bluestone CD, eds. Evidence-based Otitis Media. 2nd ed. Chicago: BC Decker, 2003:147-162

5. Acuin J. Chronic Suppurative Otitis Media: Burden of Illness and Management Options. Geneva: WHO, 2004 http://www.who.int/pbd/deafness/activities/hearing_care/otitis_media.pdf (accessed 17 January 2011).

6. Morris PS, Leach AJ. Acute and chronic otitis media. Pediatr Clin North Am 2009;56(6):1383-1399. [http:// dx.doi.org/10.1016/.jpcl.2009.09.007]

7. Miziara ID, Weber R, Araújo Filho BC, Pinheiro Neto CD. Otitis media in Brazilian human immunodeficiency Miziara ID, Weber R, Araújo Filho BC, Pinheiro Neto CD. Otitis media in Brazilian human immunodeficiency
virus infected children undergoing antiretroviral therapy. J Laryngol Otol 2007;121(11):1048-1054. [http:// dx.doi.org/10.1017/S0022215107006093]

8. United Nations Children's Fund. Towards an AIDS-free Generation: Children and AIDS: Sixth Stocktaking Report, 2013. New York: UNICEF, 2013. http://www.unaids.org/en/media/unaids/contentassets/documents unaidspublication/2013/20131129_stocktaking_report_children_aids_en.pdf (accessed 7 January 2013).
9. Joint United Nations Programme on HIV/AIDS. Global Report: UNAIDS Report on the Global AIDS Epidemic 2013. Geneva: UNAIDS, 2013. http://www.unaids.org/en/media/unaids/contentassets/documents/ epidemiology/2013/gr2013/UNAIDS_Global_Report_2013_en.pdf (accessed 17 December 2012).

10. Halama A, Voogt GR, Musgrave GM, van der Merwe CA. Prevalence of otitis media in a Venda village. S Afr Med J 1987;71(9):577-579.

11. Prescott CAJ, Kibel MA. Ear and hearing disorders in rural grade 2 (Sub B) schoolchildren in the western Cape S Afr Med J 1991;79(2):90-93.

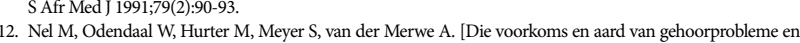
middeloorpatologiee by'n groep swart stedelike kinders in graad I]. S Afr J Commun Disord 1988;35:25-29.

13. Lee D-H, Yeo S. Clinical diagnostic accuracy of otitis media with effusion in children, and significance of myringotomy: Diagnostic or therapeutic? J Korean Med Sci 2004;19(5):739-743. [http://dx.doi.org/10.3346/ jkms.2004.19.5.739]

14. Carruthers J. Dainfern and Diepsloot: Environmental justice and environmental history in Johannesburg South Africa. Environ Justice 2008;1(3):121-126. [http://dx.doi.org/10.1089/env.2008.0526]

15. Bluestone $\mathrm{CD}$, Gates $\mathrm{GA}$, Klein JO, et al. Definitions, terminology, and classification of otitis media. Ann Otol Rhinol Laryngol 2002;111:8-18.

16. Mahadevan M, Navarro-Locsin G, Tan HKK, et al. A review of the burden of disease due to otitis media in the Asia-Pacific. Int J Pediatr Otorhinolaryngol 2012;76(5):623-635. [http://dx.doi.org/ 10.1016/j. ijporl.2012.02.031]

17. Gauteng Department of Health. Annual report 2012/13. Pretoria: Gauteng DoH, 2013. http://www gautengonline.gov.za/Publications\%20and\%20Reports/FINAL\%20Annual\%20Report\%200OP.pdf (accessed 21 December 2013).

18. Shapiro NL, Novelli V. Otitis media in children with vertically-acquired HIV infection: The Great Ormond Street Hospital experience. Int J Pediatr Otorhinolaryngol 1998;45(1):69-75. [http://dx.doi.org/10.1016/S0165$5876(98) 00089-5$

19. Tiedt NJ, Butler IRT, Hallbauer UM, et al. Paediatric chronic suppurative otitis media in the Free State Province Clinical and audiological features. S Afr Med J 2013;103(7):467-470. [http://dx.doi.org/10.7196/SAMJ.6636]

20. Swart SM, Lemmer R, Parbhoo JN, Prescott CA. A survey of ear and hearing disorders amongst a representative sample of grade 1 schoolchildren in Swaziland. Int J Pediatr Otorhinolaryngol 1995;32(1):23-34. [http://dx.doi org/10.1016/0165-5876(94)01109-B]

21. Chadha SK, Sayal A, Malhotra V, Agarwal AK. Prevalence of preventable ear disorders in over 15000 schoolchildren in northern India. I Laryngol Otol 2013;127(1):28-32. [http://dx.doi.org/ 10.1017/ S0022215112002691]

22. Green RJ. Allergic rhinitis in South African children: There is something new in the air. S Afr Fam Pract 2005;47(6):28-31.

23. Rosenfeld RM, Kay D. Natural history of untreated otitis media. Laryngoscope 2003;113(10):1645-1657. [http://dx.doi.org/10.1097/00005537-200310000-00004]

Accepted 17 January 2014. 